\title{
Use of Contemporary Groups in the Construction of Multi-Environment Trial Datasets for Selection in Plant Breeding Programs
}

\author{
Alison Smith ${ }^{1 *}$, Aanandini Ganesalingam ${ }^{2 \dagger}$, Christopher Lisle ${ }^{1}$, Gururaj Kadkol ${ }^{3}$, \\ Kristy Hobson ${ }^{3}$ and Brian Cullis ${ }^{1}$ \\ ${ }^{1}$ Centre for Bioinformatics and Biometrics, School of Mathematics and Applied Statistics, National Institute for Applied \\ Statistics Research Australia, University of Wollongong, Wollongong, NSW, Australia, ${ }^{2}$ Centre for Bioinformatics and \\ Biometrics, School of Mathematics and Applied Statistics, National Institute for Applied Statistics Research Australia, \\ University of Wollongong, Wollongong, NSW, Australia, ${ }^{3}$ New South Wales Department of Primary Industries, Tamworth \\ Agricultural Institute, Calala, NSW, Australia
}

OPEN ACCESS

Edited by:

Soren K. Rasmussen,

University of Copenhagen, Denmark

Reviewed by:

Irwin Goldman,

University of Wisconsin-Madison,

United States

Therese Bengtsson,

Swedish University of Agricultural

Sciences, Sweden

*Correspondence:

Alison Smith

alismith@uow.edu.au

${ }^{\dagger}$ Present address: Aanandini Ganesalingam, InterGrain Pty Ltd., Bibra Lake, WA,

Australia

Specialty section: This article was submitted to

Plant Breeding,

a section of the journal

Frontiers in Plant Science

Received: 30 October 2020 Accepted: 30 December 2020

Published: 02 February 2021

Citation:

Smith A, Ganesalingam A, Lisle C, Kadkol G, Hobson K and Cullis $B$ (2021) Use of Contemporary Groups in the Construction of Multi-Environment Trial Datasets for Selection in Plant Breeding Programs.

Front. Plant Sci. 11:623586.

doi: 10.3389/fp/s.2020.623586
Plant breeding programs use multi-environment trial (MET) data to select superior lines, with the ultimate aim of increasing genetic gain. Selection accuracy can be improved with the use of advanced statistical analysis methods that employ informative models for genotype by environment interaction, include information on genetic relatedness and appropriately accommodate within-trial error variation. The gains will only be achieved, however, if the methods are applied to suitable MET datasets. In this paper we present an approach for constructing MET datasets that optimizes the information available for selection decisions. This is based on two new concepts that characterize the structure of a breeding program. The first is that of "contemporary groups," which are defined to be groups of lines that enter the initial testing stage of the breeding program in the same year. The second is that of "data bands," which are sequences of trials that correspond to the progression through stages of testing from year to year. MET datasets are then formed by combining bands of data in such a way as to trace the selection histories of lines within contemporary groups. Given a specified dataset, we use the A-optimality criterion from the model-based design literature to quantify the information for any given selection decision. We demonstrate the methods using two motivating examples from a durum and chickpea breeding program. Datasets constructed using contemporary groups and data bands are shown to be superior to other forms, in particular those that relate to a single year alone.

Keywords: multi-environment trials, linear mixed models, model-based design, contemporary groups, selection, plant breeding

\section{INTRODUCTION}

Plant breeding is a process that consists of methods for the creation, selection, and fixation of superior plants in terms of productivity or quality (Moose and Mumm, 2008). During this process, the ability to select the best lines and discard others is critical in constantly improving the breeding gene pool (Zamir, 2001). Generally, breeding programs have cycle lengths that span 8 to 10 years; that is from initial cross to variety commercialization. The majority of programs follow the modified pedigree breeding method, where traits with high heritability are selected first, 
and traits of lower heritability selected in later generations when lines become fixed (Collard and Mackill, 2008). The early population stages in such programs are often focused on several traits of commercial importance, including disease resistance, herbicide tolerance, phenology type, and functional grain quality. At the advanced evaluation stages selection is focused on grain yield across target production environments (TPE), to appropriately gauge genotype by environment interaction $(\mathrm{G} \times \mathrm{E})$, as genotypes vary in their response to different environments. Note that in this paper the terms "genotype" and "line" are used synonymously.

While there are multiple traits of interest under selection, yield is often the trait of foremost interest. In order to achieve efficiency in yield selection, yield data is generated from a series of field trials across years (synonymous with seasons) and geographical locations, known as multi-environment trials (METs). METs are an essential evaluation tool in plant breeding programs, as they enable an effective measure of $\mathrm{G} \times \mathrm{E}$. This is particularly important in the Australian agricultural environment, which is known to be extremely variable between locations and seasons (Chapman et al., 2003). As a result, advanced evaluation stages in breeding programs use expanded numbers of evaluation environments to appropriately assess across all TPE.

The breeding process can be viewed as a multi-year, multicycle collection of data (Arief et al., 2019). In particular, we find that programs are underpinned by a grouping of lines that are derived together from a fixed number of crosses at the crossing block stage. These lines are essentially "born" together and are subsequently tested together. This cohort of lines then progress through the bulk population stages, are derived to fixed lines and tested sequentially in the advanced evaluation stages. We refer to these broad grouping of lines as "contemporary groups" (CGs). As an example we consider the Durum Breeding Australia (DBA) program from one of our motivating examples (see section 3) in which there are four stages of testing (denoted S1 to S4). Within the time-frame 2015 to 2018, the following four CGs were created: CG15, CG16, CG17, and CG18 corresponding to lines in S1 trials in those years. Then, for example, a subset of the lines from CG15 was progressed to S2 trials in 2016 and so on to S3 in 2017 and finally to S4 in 2018. As lines progress through stages they decrease in number, similar to the narrowing observed in the funnel structure seen in Figure 1. Following on from this figure, four selection decisions would be made annually on S1, S2, S3, and S4 lines as they progress to the next stage of testing. The final stage selection decision, that is, for S4, is concerned with the submission of elite lines to the Australian national crop variety testing program, the National Variety Trials (NVT).

For plant breeding programs in Australia, the preferred approach for the analysis of MET data is the single stage Factor Analytic Linear Mixed Model (FALMM) of Smith et al. (2001), with modeling of spatial variation for individual trials (Gilmour et al., 1997; Stefanova et al., 2009). This analysis approach is also the preferred method of analysis for the NVT (Smith and Cullis, 2018). Oakey et al. (2007) extended these models with the inclusion of information on genetic relatedness through ancestral information (pedigree), which Beeck et al. (2010) utilized to

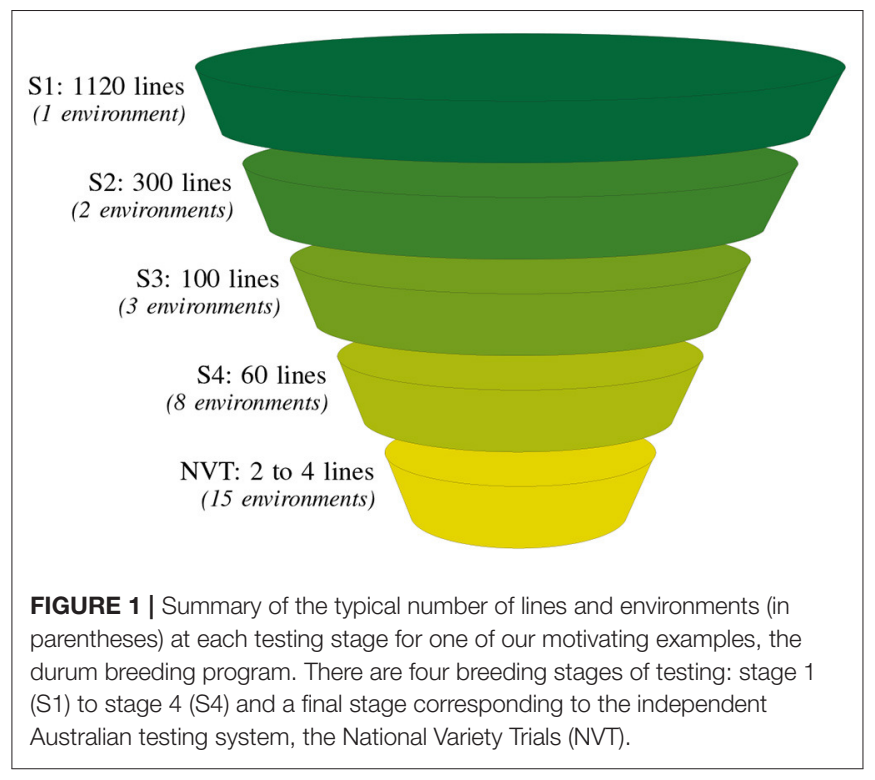

enable partitioning of additive and non-additive effects for selection in a canola breeding program.

There is a substantial amount of literature demonstrating the improved selection accuracy resulting from the use of a one stage FALMM that incorporates pedigree (ancestry) information (see Oakey et al., 2007; Beeck et al., 2010; Smith and Cullis, 2018; Ukrainetz et al., 2018). The inclusion of pedigree information via the numerator relationship matrix (NRM) provides links between genotypes both within and between environments. This enables more reliable estimation of genetic variance parameters and thence more accurate predictions of total genetic effects as required for selection.

It is well-known that these developments in methods of analysis contribute to genetic gains, as shown by the wide-spread adoption of the FALMM of Smith et al. (2001) in Australian plant breeding programs (Gogel et al., 2018). In Table 1, we have compiled a concise summary of literature specific to plant breeding METs and the methods of analysis. In the process of compiling this summary, we noted that there are very few papers written on the construction of the underlying MET datasets to realize these genetic gains from an analysis point of view. For example, the paper by Arief et al. (2019) indicates that a MET dataset across stages of a breeding program in a combined analysis is the most advantageous. However, they used a two-stage linear mixed model without the inclusion of pedigree information. In contrast, Yan and Rajcan (2003) used a similar approach (single stage, without pedigree) and found that a single year MET dataset was sufficient to identify the best and worst selections. Despite the vast amounts of data breeding programs generate, it is clear that there is little consensus on the construction of datasets for selection decisions in the current literature.

The inherent structure of the breeding program (stages within a year) and thus selection on a stage basis often results in the analysis of plant breeding datasets comprising a series of single 
TABLE 1 | Summary of studies based on plant breeding METs, their dataset composition, and methods of analysis.

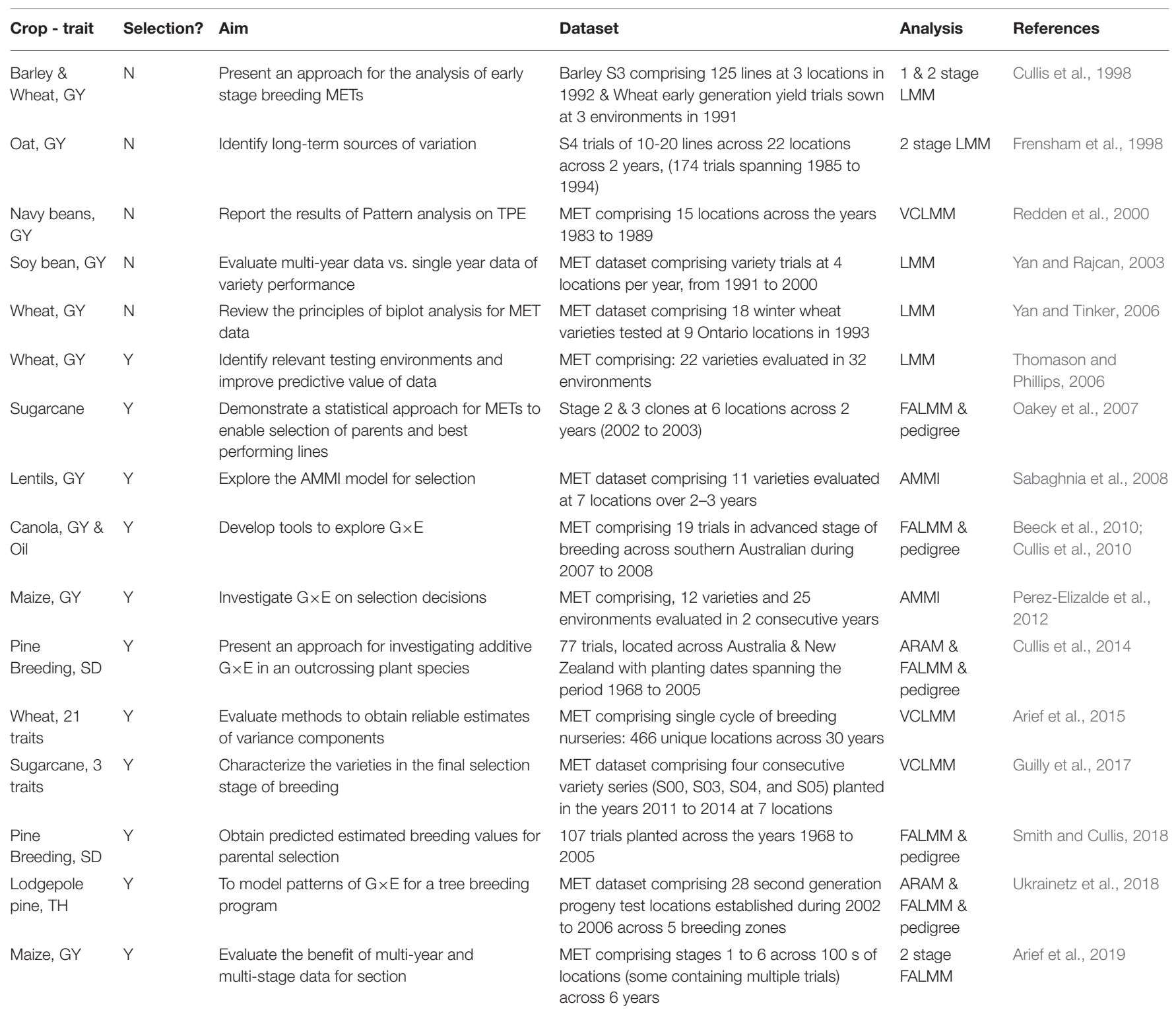

The Selection column indicates if the aim of the paper was selection on the given dataset. GY, Grain Yield; SD, Stem Diameter; TH, Tree Height; AMMI, Additive Main effect and Multiplicative Interaction; ARAM, Approximate Reduced Animal Model; FALMM, Factor Analytic Linear Mixed Model; LMM, Linear Mixed Model; VCLMM, Variance Component Linear Mixed Model.

year based analyses (Arief et al., 2019). Bernal-Vasquez et al. (2017) finds similarly that breeding program datasets are often analyzed on a year basis and not over years, due to the reasons: that it is simpler/faster and that it is difficult to estimate variation across years due to the lack of common lines between breeding stages. However, due to the commercial nature of plant breeding programs, there is limited literature on the dataset construction of plant breeding METs, within the context of a commercial breeding operation.

One of the central aims of plant breeding programs is to select high yielding and stable genotypes across environments. This is why plant breeding programs evaluate lines over a large number of locations and years as it enables estimates of random occurring cycles of normal and extreme conditions in the TPE (Rosielle and Hamblin, 1981). In the Australian context large and complex $\mathrm{G} \times \mathrm{E}$ has been reported specifically for wheat breeding (Bänziger and Cooper, 2001). For barley, wheat, oats, lupins, peas, lentils, and canola Cullis et al. (2000) found that the main sources of $\mathrm{G} \times \mathrm{E}$ are what they define as "non-static", that is linked to seasonal influences. This alone contributed to $41 \%$ of the total variance. Frensham et al. (1998) similarly found with a Southern Australian oat breeding program that the genotype by year by location $(\mathrm{G} \times \mathrm{Y} \times \mathrm{L})$ variance component accounted for $41.1 \%$ of the total phenotypic variance. Arief et al. (2015) summarized multiple plant breeding studies, finding that the $\mathrm{G} \times \mathrm{Y} \times \mathrm{L}$ variance component as a proportion of total phenotypic 
variance was $29 \%$ for wheat and $25 \%$ for navy beans breeding programs in Australia. Therefore, there is a need for multiple years of data to accurately quantify genotype performance in the presence of substantial $\mathrm{G} \times \mathrm{E}$ in order to make accurate selection decisions. This is clearly not addressed by single year/single stage based data analysis in a plant breeding program.

The aim of this paper is to demonstrate the utilization of the CG concept for dataset construction. The basic premise is to include sufficient trials to optimize the amount of data on the lines under consideration for selection. By tracing CGs across stages and years it is possible to form a MET dataset with the desired properties. In order to quantify the impact of this approach we use the A-optimality criterion from modelbased design theory. The paper is arranged as follows. Section 2 outlines the methodology for MET dataset construction using CGs. The use of A-optimality for comparing datasets is described. In section 3 the methods are applied to two motivating examples from Australian plant breeding programs. Some concluding remarks are given in section 4.

\section{METHODS FOR MET DATASET CONSTRUCTION}

The CG concept for MET data construction is first illustrated using a hypothetical breeding program with four stages of testing (S1 to S4) and in which lines progress through stages without fasttracking (skipping stages) or retention (remaining in a stage for more years of testing). The aim is to construct a dataset to enable accurate selection decisions for 2018. First consider the decisions on lines in S4 in 2018. These lines commenced their testing in S1 trials in 2015 (so are all members of CG15), were selected to be tested in S2 trials in 2016, then S3 trials in 2017, and finally S4 trials in 2018. Thus, in order to capture all of the data on the lines under consideration for selection, we would combine data from all of the trials in this sequence. Overall, this would suggest a separate analysis for each of the selection decisions, based on combining data from the following trials:

- Selection decision S1: S1 trials 2018

- Selection decision S2: S2 trials $2018+$ S1 trials 2017

- Selection decision S3: S3 trials 2018 + S2 trials $2017+$ S1 trials 2016

- Selection decision S4: S4 trials $2018+$ S3 trials $2017+$ S2 trials $2016+$ S1 trials 2015 .

It is instructive to illustrate this compilation of trials across stages and years using tables such as Table 2. In this table the diagonal bands of stages across years are labeled as A to I, with the labels A to D being assigned in such a way that they align with the S1 to S4 trials in the year of selection (here 2018). The datasets described above correspond to the diagonal bands of trials labeled as A to D. Thus, for example, band D comprises data from S1 trials in 2015, S2 trials in 2016, S3 trials in 2017, and S4 trials in 2018. It is important to note that, for any given trial, the data from all of the harvested plots is included and not just the data on the lines of interest. Thus, for example, the data from S1 trials in 2015 relates to all of the lines tested in those trials. We describe bands $\mathrm{A}$ to $\mathrm{F}$
TABLE 2 | Data bands for potential inclusion in a MET dataset for selection decisions in 2018 from a breeding program with four stages of selection (S1 to S4).

\begin{tabular}{lllllll}
\hline & \multicolumn{6}{c}{ Year } \\
\cline { 2 - 7 } Stage & $\mathbf{2 0 1 3}$ & $\mathbf{2 0 1 4}$ & $\mathbf{2 0 1 5}$ & $\mathbf{2 0 1 6}$ & $\mathbf{2 0 1 7}$ & $\mathbf{2 0 1 8}$ \\
\hline S1 & F & E & D & C & B & A \\
S2 & G & F & E & D & C & B \\
S3 & H & G & F & E & D & C \\
S4 & I & H & G & F & E & D \\
\hline
\end{tabular}

Data bands correspond to diagonal sequences of stages across years and are labeled as A to 1 .

in Table 2 as being "complete" in the sense that they trace back to the first stage of testing, namely S1. In contrast, bands G to I are incomplete, with band $\mathrm{G}$ missing $\mathrm{S} 1$ trials, band $\mathrm{H}$ missing S1 and S2 trials and band I missing S1, S2, and S3 trials. This has implications in terms of selection bias which will be discussed in section 3.

In the absence of retention or fast-tracking, all the lines in S1, S2, S3, and S4 in 2018 are members of CG18, CG17, CG16, and CG15, respectively. Thus, all the lines within a stage in 2018 belong to a single CG only and the entire selection history for any of these lines is captured in the associated data band. The generalization to more complex scenario will be discussed in the context of the motivating examples (see section 3 ).

In terms of information available for each of the four selection decisions it is instructive to differentiate between "direct" and "indirect" information. The former relates to observed data so is maximized by including all trials in which the lines of interest have been grown. In the hypothetical example this corresponds to the bands so suggests the conduct of four analyses each based on a separate band (A, B, C, and D). However, the use of a FALMM for analysis creates the possibility of also using indirect information derived from genetically related lines in other trials. We would therefore recommend undertaking a single analysis using data combined across these bands. This recommendation can be justified by applying the method described in section 2.1 to quantify information for selection. Finally, we note that in the MET analysis, $\mathrm{G} \times \mathrm{E}$ is modeled with reference to environments which are defined to be combinations of trial locations and years. Combining across bands may lead to the presence of multiple trials at a single location within a year. For example, in any given year, locations with S1 trials also typically include S2, S3, and S4 trials. We refer to such trials as "co-located." This will be discussed further in section 3.

\subsection{Quantifying Information for Selection in MET Datasets}

In order to discriminate among possible MET datasets in terms of the amount of information available for selection decisions, we note that the problem has strong links with optimal (modelbased) design. As Butler et al. (2014) state, "The goal of optimal design is to discriminate among competing designs in an effort to maximize the treatment information from a fixed 
number of experimental units." This requires the use of an optimality criteria, and, in the context of plant breeding trials in which the treatments are genotypes and the aim is selection, the A-optimality criteria is appropriate since this aligns with minimizing the probability of an incorrect selection decision (Bueno Filho and Gilmour, 2003). A-optimality is based on the so-called A-value which is the average pairwise variance of elementary treatment contrasts. We therefore propose to use Avalues to quantify the treatment (genotype) information available in any given MET dataset.

In model-based design, A-values are computed under a prespecified Linear Mixed Model (LMM) which we will term the design model. Specification of the design model requires specification of the fixed and random effects, the variance models for the random effects and residuals and the values of the associated variance parameters. The design model is usually chosen to be as close as possible to that expected for the analysis. Additionally, the variance parameter values are chosen as being "typical" so may be based on historic analyses. The model proposed in this paper for the analysis of MET data is the FALMM with the inclusion of pedigree information. Genotype selections using this model are typically focused on the measure of overall genotype performance (across environments) as presented in Smith and Cullis (2018). However, the factor analytic variance parameters are specific to the individual environments in the dataset so that typical values do not exist. Therefore a more generic, but still realistic design model is required for assessing MET dataset information. We have chosen a variance component model that involves random genotype main effects and random $\mathrm{G} \times \mathrm{E}$ effects, both of which are partitioned into additive and non-additive effects. This is, in fact, a sub-model of the FALMM. The A-values are then computed for the total (additive plus non-additive) genotype main effects since these provide a measure of average performance of genotypes across environments.

In order to determine reasonable values for the variance parameters in this design model we consider Cullis et al. (2000) who conducted variance component analyses of grain yield in 22 MET datasets from Australian crop variety evaluation programs. The environments in those datasets were classified according to the year, the geographic region and possibly location within region so Cullis et al. (2000) partitioned $\mathrm{G} \times \mathrm{E}$ accordingly. In our motivating examples we do not have regional information nor are trials typically located in identical positions from year to year. However, we recognize the importance of genotype by year interaction so maintain this as a separate source in the design model. Thus we have used the genotype main effects $(\mathrm{G})$, genotype by year interaction $(\mathrm{G} \times \mathrm{Y})$, and Error sources of variation from Cullis et al. (2000), and have added together the remaining sources to form residual genotype by environment interaction. The mean percentage contributions for each of these sources across all 22 datasets was 13.77\% (G),

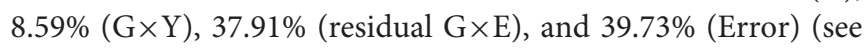
Table 3). In the model-based design literature, and without loss of generality, a value of one is typically assumed for the error variance. We adopt the same approach here (see second row in Table 3).
TABLE 3 | Variance parameter values for design model for genotype main effects $(G)$, genotype by year interaction $(G \times Y)$, residual genotype by environment interaction $(\mathrm{G} \times \mathrm{E})$, and Error.

\begin{tabular}{lcccc}
\hline & G & GxY & GxE & Error \\
\hline Mean \% from Cullis et al. (2000) & 13.77 & 8.59 & 37.91 & 39.73 \\
Total variance parameter & 0.35 & 0.22 & 0.95 & 1.00 \\
Additive variance parameter & $0.28 / \bar{a}$ & $0.18 / \bar{a}$ & $0.76 / \bar{a}$ & \\
Non-additive variance parameter & 0.07 & 0.04 & 0.19 &
\end{tabular}

Rows in the table are: means of percentages in Cullis et al. (2000); associated (total) variance parameter values assuming error variance of one; additive variance parameter values (numerator is $80 \%$ of total values and denominator, $\bar{a}$, is the mean of the diagonal elements of NRM); non-additive variance parameter values (20\% of total values).

In contrast to our approach, the analyses in Cullis et al. (2000) do not involve information on genetic relatedness. We therefore make the further assumption that additive variance comprises $80 \%$ of total variance. This represents an average from the analyses of numerous Australian plant breeding datasets. The final values for the variance parameters in the design model are given in the third and fourth rows of Table 3. All A-values in this paper were computed using ASReml-R (Butler et al., 2017). The code is provided in Appendix A.

\section{RESULTS}

In this section we show the application of the methods presented in section 2 to two motivating examples.

\subsection{Durum Breeding Program}

Durum wheat (Triticum durum desf.) breeding in Australia is currently resourced on agronomic zones of production and funded by New South Wales Department of Primary Industries (NSW DPI), The University of Adelaide and the Grains Research and Development Corporation (GRDC) under the Durum Breeding Australia (DBA) project. The motivating example of our paper is the DBA North program that operates out of Tamworth Agricultural Institute, capturing TPEs in New South Wales (NSW) up to and including central Queensland (QLD). The structure of the program is illustrated in Figure 1. The S1 in any year contains on average 1120 lines evaluated in one or two environments. As the program progresses the line numbers decrease to on average, 60 lines in the S4, evaluated in eight environments.

The aim of this paper is to construct a dataset to evaluate the performance of the 2018 S1, S2, S3, and S4 lines for selection and progression to the next stage of testing. The data available for this purpose spanned the period 2013 to 2018 so the sequences of stages and years (and thence data bands) are the same as shown in Table 2. The actual numbers of trials in each stage and year, and the total numbers of trials in each complete data band, are given in Table 4. Note that there were co-located trials within stages in many environments so that the numbers of environments is also provided in this table. The numbers of test lines for each stage and year (2013 to 2018) are given in Table 5. Note that test lines refer only to the lines under consideration for selection, so excludes 
check varieties, for example. At any stage of selection, a line may be selected to progress to the next stage of testing, retained in the same stage or rejected. In contrast to the hypothetical example, lines are often retained within later stages for additional year/s of testing. Retentions may occur due to limitations in seed production, or even a holding pattern while awaiting disease and/or quality data. This has resulted in the lines in later stages comprising a mixture of CGs. The distribution across CGs for 2018 lines are given as the final columns in Table 5. For example, the majority (66) of lines in S3 in 2018 correspond to CG16 so have followed the simple progression along band $\mathrm{C}$ (that is, they progressed from S1 trials in 2016 to S2 trials in 2017 to S3 trials in 2018). But a fair number (22) correspond to CG15 and progressed from S1 trials in 2015 to S2 trials in 2016 to S3 trials in 2017 and were then retained in S3 in 2018. Finally, five lines correspond to CG14 and progressed from S1 trials in 2014 to S2 trials in 2015 to S3 trials in 2016 and were then retained in S3 in 2017 and 2018. This has implications for construction of the MET dataset.

The starting point for MET dataset construction for selection decisions on the 2018 lines (S1 to S4) involves all trials in bands A-D as described in the hypothetical example. With the retention of lines it is clear that this would fail to capture much of the data on the 30 S3 and S4 lines in 2018 that belonged to CG14 and CG13 (see Table 5). For example, Table 6 shows there are nine lines in S4 for which 5 years of data would be missing if the dataset comprised only bands A-D; another nine lines for which 4 years would be missing and a further seven lines missing 2 or 3 years of data. We believe this is unacceptable. We therefore

TABLE 4 | Number of trials and environments (presented as trials/environments) in each stage (S1 to S4) and year (2013-2018) in the durum breeding program with data bands indicated as superscripts.

\begin{tabular}{|c|c|c|c|c|c|c|c|}
\hline \multirow[b]{2}{*}{ Stage } & \multicolumn{6}{|c|}{ Year } & \multirow{2}{*}{$\begin{array}{c}\text { Band } \\
\text { Totals }\end{array}$} \\
\hline & 2013 & 2014 & 2015 & 2016 & 2017 & 2018 & \\
\hline S1 & $2 / 1^{F}$ & $5 / 2^{E}$ & $6 / 1^{D}$ & $6 / 1^{C}$ & $7 / 1^{B}$ & $6 / 1^{A}$ & $6 / 1^{A}$ \\
\hline S2 & $6 / 3^{G}$ & $8 / 2^{F}$ & $8 / 2^{E}$ & $3 / 1^{D}$ & $3 / 1^{C}$ & $6 / 2^{B}$ & $13 / 3^{B}$ \\
\hline S3 & $4 / 4^{H}$ & $3 / 3^{G}$ & $5 / 4^{F}$ & $3 / 3^{E}$ & $3 / 3^{D}$ & $3 / 3^{C}$ & $12 / 5^{C}$ \\
\hline \multirow[t]{3}{*}{ S4 } & $9 / 9^{\prime}$ & $8 / 8^{H}$ & $12 / 10^{G}$ & $6 / 5^{F}$ & $11 / 10^{E}$ & $6 / 6^{D}$ & $18 / 11^{D}$ \\
\hline & & & & & & & $27 / 17^{E}$ \\
\hline & & & & & & & $21 / 12^{F}$ \\
\hline
\end{tabular}

The final column gives the total numbers of trials and environments in each of the complete data bands ( $A$ to $F$ ). investigate the addition of bands $\mathrm{E}$ and $\mathrm{F}$ to the data. Table 6 shows the improvement in capturing more of the data on the lines of interest. Full data on the lines of interest could be obtained by adding band $G$ but we caution against this because band $G$ is incomplete (it is missing S1 trials, see Table 2) so we do not have the entire selection history for many of the lines in band $G$. The inclusion of band $G$, or indeed the other incomplete bands $\mathrm{H}$ and I (so that the entire rectangle of data is included) may result in "selection bias," that is, bias in the estimates of the genetic variance parameters (Thompson, 1973) so is not recommended. The final dataset is therefore chosen to comprise bands A-F. With this dataset only five lines under scrutiny for selection (in S4) are missing data and the amount missing is small ( 1 or 2 years out of a total of 6 years).

The final MET dataset (bands A-F) for analysis comprised yield data on 6,951 lines from 21,660 plots corresponding to 97 trials (see Table 4) across 30 environments. Each field trial was sown as a rectangular array indexed by field rows and columns. Trials were sown in a serpentine sequence and harvested in the row direction with all other management regimes applied via pathways in the column dimension. Trials were designed as grid-plot, partially replicated ( $p$-rep) (Cullis et al., 2006) or randomized complete block designs, with two to three replicates. Summary information for the 30 environments is given in

TABLE 6 | MET dataset construction for 2018 selection decisions in durum breeding program.

\begin{tabular}{|c|c|c|c|c|c|}
\hline \multirow[b]{2}{*}{ Stage } & \multirow{2}{*}{$\begin{array}{l}\text { \# years } \\
\text { missing }\end{array}$} & \multicolumn{4}{|c|}{ Bands in dataset } \\
\hline & & A-D & A-E & $A-F$ & A-G \\
\hline S1 & 0 & 1148 & 1148 & 1148 & 1148 \\
\hline S2 & 0 & 315 & 315 & 315 & 315 \\
\hline \multirow[t]{2}{*}{ S3 } & 0 & 88 & 93 & 93 & 93 \\
\hline & 2 & 5 & 0 & 0 & 0 \\
\hline \multirow[t]{6}{*}{ S4 } & 0 & 31 & 42 & 51 & 56 \\
\hline & 1 & 0 & 1 & 3 & 0 \\
\hline & 2 & 6 & 0 & 2 & 0 \\
\hline & 3 & 1 & 0 & 0 & 0 \\
\hline & 4 & 9 & 13 & 0 & 0 \\
\hline & 5 & 9 & 0 & 0 & 0 \\
\hline
\end{tabular}

Number of lines missing years of data in datasets comprising bands $A-D, A-E, A-F$, and A-G. Total number of lines for selection: 1148, 315, 93, and 56 (for stages S1 to S4).

TABLE 5 | Number of test lines in each stage (S1 to S4) and year (2013-2018) in the durum breeding program.

\begin{tabular}{|c|c|c|c|c|c|c|c|c|c|c|c|c|}
\hline \multirow[b]{2}{*}{ Stage } & \multicolumn{6}{|c|}{ Number of test lines } & \multicolumn{6}{|c|}{ Number of 2018 test lines } \\
\hline & 2013 & 2014 & 2015 & 2016 & 2017 & 2018 & CG18 & CG17 & CG16 & CG15 & CG14 & CG13 \\
\hline S1 & 582 & 1485 & 1000 & 1163 & 1303 & 1148 & 1148 & 0 & 0 & 0 & 0 & 0 \\
\hline S2 & 105 & 361 & 413 & 388 & 379 & 315 & 0 & 315 & 0 & 0 & 0 & 0 \\
\hline S3 & 30 & 92 & 92 & 92 & 90 & 93 & 0 & 0 & 66 & 22 & 5 & 0 \\
\hline S4 & 25 & 41 & 57 & 55 & 53 & 56 & 0 & 0 & 0 & 31 & 12 & 13 \\
\hline
\end{tabular}

The final columns give the number of lines in each contemporary group (CG18-CG13) for lines under consideration for selection in 2018. 
Table 7. There were 15 environments with co-located trials, ranging in number from two to 13 . The co-located trials related either to different stages or to multiple trials within stages (also see Table 4), the latter being due to physical restrictions on trial sizes. We note that co-located trials are only deemed to comprise a single environment when they are all managed in the same way, that is, they are sown and harvested within a similar time frame and subjected to the same agronomy practices including fertilizer, herbicide, and pathway regimes.

The pedigree information associated with the above trial data contained 7,628 records. All lines in the MET data set had pedigree information. This was the first time a pedigree file had been created for this breeding program and included

TABLE 7 | Summary of environments in the durum MET dataset: number of trials for each stage of testing (S1, S2, S3, S4) and total number of trials.

\begin{tabular}{|c|c|c|c|c|c|c|c|c|}
\hline \multirow[b]{2}{*}{ Environment } & \multicolumn{5}{|c|}{ Number of trials } & \multirow[b]{2}{*}{ nplot } & \multirow[b]{2}{*}{ nline } & \multirow{2}{*}{$\begin{array}{l}\text { Mean } \\
\text { yield }\end{array}$} \\
\hline & S1 & S2 & S3 & S4 & Total & & & \\
\hline 2013-Breeza & 2 & 0 & 0 & 0 & 2 & 720 & 585 & 2.88 \\
\hline 2014-Breeza & 3 & 0 & 0 & 0 & 3 & 1296 & 937 & 4.28 \\
\hline 2014-Edgeroi & 0 & 4 & 0 & 0 & 4 & 768 & 364 & 2.78 \\
\hline 2014-Tworth & 2 & 4 & 0 & 0 & 6 & 1468 & 915 & 3.93 \\
\hline 2015-Breeza & 0 & 0 & 2 & 0 & 2 & 384 & 96 & 5.15 \\
\hline 2015-Edgeroi & 0 & 4 & 1 & 0 & 5 & 1056 & 498 & 1.83 \\
\hline 2015-Nstar & 0 & 0 & 1 & 0 & 1 & 192 & 96 & 5.05 \\
\hline 2015-Tworth & 6 & 4 & 1 & 0 & 11 & 2244 & 1499 & 4.00 \\
\hline 2016-Breeza & 0 & 0 & 1 & 1 & 2 & 372 & 152 & 4.35 \\
\hline 2016-Edgeroi & 0 & 0 & 0 & 1 & 1 & 180 & 60 & 4.79 \\
\hline 2016-Gurley & 0 & 0 & 0 & 1 & 1 & 180 & 60 & 5.62 \\
\hline 2016-Nstar & 0 & 0 & 1 & 2 & 3 & 552 & 152 & 5.49 \\
\hline 2016-Tworth & 6 & 3 & 1 & 1 & 11 & 2628 & 1704 & 4.81 \\
\hline 2017-Blbgra & 0 & 0 & 0 & 1 & 1 & 180 & 60 & 1.12 \\
\hline 2017-Breeza & 0 & 0 & 1 & 1 & 2 & 384 & 158 & 5.31 \\
\hline 2017-Bribbaree & 0 & 0 & 0 & 1 & 1 & 180 & 60 & 1.20 \\
\hline 2017-Coonamble & 0 & 0 & 0 & 1 & 1 & 180 & 60 & 1.61 \\
\hline 2017-Edgeroi & 0 & 0 & 0 & 1 & 1 & 180 & 60 & 3.93 \\
\hline 2017-Garah & 0 & 0 & 0 & 1 & 1 & 180 & 60 & 1.84 \\
\hline 2017-Gurley & 0 & 0 & 0 & 1 & 1 & 180 & 60 & 2.12 \\
\hline 2017-Nstar & 0 & 0 & 1 & 1 & 2 & 384 & 158 & 3.41 \\
\hline 2017-Tworth & 7 & 3 & 1 & 2 & 13 & 3014 & 1836 & 4.26 \\
\hline 2017-Westmar & 0 & 0 & 0 & 1 & 1 & 180 & 60 & 2.24 \\
\hline 2018-Blbgra & 0 & 0 & 0 & 1 & 1 & 198 & 66 & 1.24 \\
\hline 2018-Breeza & 6 & 3 & 1 & 1 & 11 & 2502 & 1629 & 5.53 \\
\hline 2018-Coonamble & 0 & 0 & 0 & 1 & 1 & 198 & 66 & 1.55 \\
\hline 2018-Gurley & 0 & 0 & 1 & 0 & 1 & 210 & 105 & 2.23 \\
\hline 2018-Moree & 0 & 0 & 0 & 1 & 1 & 198 & 66 & 1.51 \\
\hline 2018-Trangie & 0 & 0 & 0 & 1 & 1 & 198 & 66 & 1.02 \\
\hline 2018-Tworth & 0 & 3 & 1 & 1 & 5 & 1074 & 481 & 2.24 \\
\hline
\end{tabular}

Number of plots (nplot) and lines (nline); mean yield (t/ha). in the analysis. This was a significant undertaking as the durum breeding program was established in the 1960's and a comprehensive pedigree file (outside annual crossing block information) had not existed in the program since this time. The NRM was formed using the pedicure package (Butler, 2019) in R (R Development Core Team, 2015). The inbreeding coefficients of lines with phenotypic data ranged from 0.750 to 0.998 with mean of 0.905 .

Finally, the approach described in section 2.1 for comparing MET datasets in terms of the information for selection was applied for each stage of selection and for three types of MET dataset, namely the 2018 data for each stage, the diagonal band of data for each stage and the final dataset (bands A-F). Thus, for S4 selections, the three datasets comprised data from S4 trials in 2018 alone; data from trials in band D (S4 trials in $2018+$ S3 trials in $2017+$ S2 trials in $2016+$ S1 trials in 2015) and the final dataset. For S3 selections, the three datasets comprised data from S3 trials in 2018 alone; data from trials in band C (S3 trials in $2018+$ S2 trials in $2017+$ S1 trials in 2016) and the final dataset. For S2 selections, the three datasets comprised data from S2 trials in 2018 alone; data from trials in band B (S2 trials in $2018+$ S1 trials in 2017) and the final dataset. Note that for S1 selections, the single year dataset ( $\mathrm{S} 1$ trials in 2018) is equivalent to the band dataset (band A). The resultant A-values are shown in Figure 2. This clearly shows the superiority of the final dataset in each case. The reduction in A-values is largely driven by an increase in the amount of direct information (as reflected in the mean numbers of environments per line) but there is also a strong contribution from indirect information. For example, the S1 lines under consideration for selection in 2018 were only grown in a single environment (see Table 4) so there is no difference in

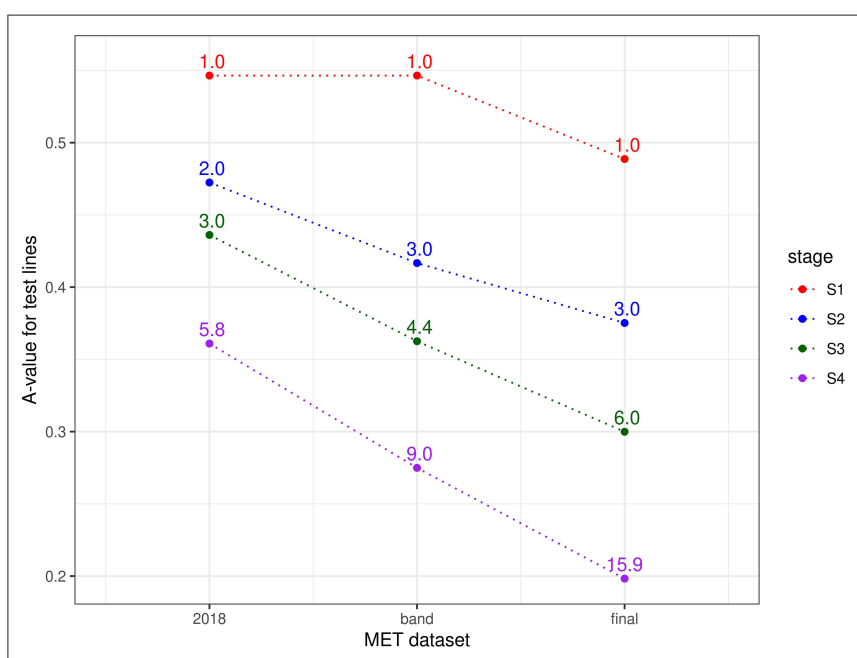

FIGURE 2 | A-values for lines under consideration for selection in durum breeding program in 2018 (stage S4: 56 lines, S3: 93 lines, S2: 315 lines, and S1: 1148 lines). A-values are given for three types of MET dataset, namely 2018 data for each stage; diagonal band of data for each stage and the final dataset constructed as in section 3.1. The points are labeled with the associated mean numbers of environments in which these lines were grown. Note that for S1 the 2018 and band datasets are the same. 
direct information between using the 2018 data alone for this stage compared with the final dataset. However, the A-value for the final dataset is much lower, indicating the impact of indirect information from relatives of the $\mathrm{S} 1$ lines.

\subsection{Chickpea Breeding Program}

In Australia, the chickpea (Cicer arietinum L.) breeding program is managed under the umbrella of Pulse Breeding Australia (PBA). PBA is an Australian government funded project through the Grains Research and Development Corporation (GRDC). PBA coordinates and funds the breeding activities of the four pulse crops of economic importance-chickpeas, field peas, faba beans, and lentils across the Australian growing environment. The chickpea program under PBA comprises three sub-programs, which are based on the following germplasm streams-desi (north and south) and kabuli. We will focus on the desi south sub program managed by NSW DPI. This program evaluates lines annually, across southern NSW, Victoria and South Australia in three stages, namely S1, S2, and S3 across multiple locations. The structure of the program is similar to that of the program illustrated in Figure 1. The S1 in any year contains on average 650 lines evaluated across five environments. As the program progresses the line numbers decrease to on average, 70 lines in S3, evaluated in eight environments.

Here we consider the MET dataset construction to evaluate the performance of test lines in all three stages in 2019. The data available for this purpose spanned the period 2016 to 2019. The sequences of stages and years (and thence data bands), together with their associated numbers of trials are given in Table 8. Note that there were no co-located trials within stages so that the numbers of environments in the cells of this table are the same as the numbers of trials. The numbers of test lines for each stage and year (2016 to 2019) are given in Table 9. The distribution across CGs for 2019 lines are given as the final columns in Table 9. Thus, for example, the majority (46) of lines in S3 correspond to CG17 (so have followed the simple progression along band C) but 11 correspond to CG16 and one to CG14.

The starting point for MET dataset construction for selection decisions on the 2019 lines (S1 to S3) involves all trials in bands A-C. However, this would fail to capture all the data on the 11 S3 lines in 2019 that belonged to CG16 and the single line that belonged to CG14 (also see Table 10). Addition of band D

TABLE 8 | Number of trials in each stage (S1 to S3) and year (2016-2019) in the chickpea breeding program with data bands indicated as superscripts.

\begin{tabular}{|c|c|c|c|c|c|}
\hline \multirow[b]{2}{*}{ Stage } & \multicolumn{4}{|c|}{ Year } & \multirow{2}{*}{$\begin{array}{l}\text { Band } \\
\text { Totals }\end{array}$} \\
\hline & 2016 & 2017 & 2018 & 2019 & \\
\hline S1 & $4^{D}$ & $5^{C}$ & $5^{B}$ & $6^{A}$ & $6^{A}$ \\
\hline S2 & $4^{E}$ & $6^{D}$ & $6^{C}$ & $7^{B}$ & $12^{B}$ \\
\hline \multirow[t]{2}{*}{ S3 } & $5^{F}$ & $8^{E}$ & $8^{D}$ & $9^{C}$ & $20^{C}$ \\
\hline & & & & & $18^{D}$ \\
\hline
\end{tabular}

The final column gives the total numbers of trials in each of the complete data bands ( $A$ to $D)$. accommodates the former and provides an additional year for the latter. Full data on the lines of interest could be obtained by adding bands $\mathrm{E}$ and $\mathrm{F}$ but once again we caution against this because these bands are incomplete so would introduces many lines with incomplete selection histories. The final dataset is therefore chosen to comprise bands A-D. With this dataset only a single line (in S3) is missing data. We note that this line has been retained in S3 for a number of years and is of less interest in terms of promotion to the next stage of testing (in the NVT).

The final MET dataset (bands A-D) for analysis comprised yield data on 2,448 lines from 18,936 plots corresponding to 56 trials (see Table 8) across 28 environments. Summary information for the 28 environments is given in Table 11. There were 18 environments with co-located trials, with between two to three trials. These were all associated with different stages within environments.

The pedigree information associated with the above trial data contained 2,983 records. All lines in the MET data set had pedigree information. The inbreeding coefficients of lines with phenotypic data ranged from 0.500 to 0.999 with mean of 0.7404 .

The information available for selection at each stage was assessed using a similar approach to the durum example, namely computing A-values for test lines for three types of MET dataset. For S3 selections, the three datasets comprised data from S3 trials in 2019 alone; data from trials in band C (S3 trials in 2019+ S2 trials in $2018+$ S1 trials in 2017) and the final dataset (trials in bands A-D). For S2 selections, the three datasets comprised data from S2 trials in 2019 alone; data from trials in band B (S2 trials

TABLE 9 | Number of test lines in each stage (S1 to S3) and year (2016-2019) in the chickpea breeding program.

\begin{tabular}{|c|c|c|c|c|c|c|c|c|c|c|}
\hline \multirow[b]{2}{*}{ Stage } & \multicolumn{4}{|c|}{ Number of test lines } & \multicolumn{6}{|c|}{ Number of 2019 test lines } \\
\hline & 2016 & 2017 & 2018 & 2019 & CG19 & CG18 & CG17 & CG16 & CG15 & CG14 \\
\hline S1 & 443 & 559 & 763 & 638 & 633 & 5 & 0 & 0 & 0 & 0 \\
\hline S2 & 113 & 100 & 146 & 176 & 0 & 176 & 0 & 0 & 0 & 0 \\
\hline S3 & 58 & 58 & 49 & 58 & 0 & 0 & 46 & 11 & 0 & 1 \\
\hline
\end{tabular}

The final columns give the number of lines in each contemporary group (CG19-CG14) for lines under consideration for selection in 2019.

TABLE 10 | MET dataset construction for 2019 selection decisions in chickpea breeding program.

\begin{tabular}{|c|c|c|c|c|c|}
\hline \multirow[b]{2}{*}{ Stage } & \multirow{2}{*}{$\begin{array}{l}\text { \# years } \\
\text { missing }\end{array}$} & \multicolumn{4}{|c|}{ Bands in dataset } \\
\hline & & $A-C$ & A-D & A-E & A-F \\
\hline S1 & 0 & 638 & 638 & 638 & 638 \\
\hline S2 & 0 & 176 & 176 & 176 & 176 \\
\hline \multirow[t]{4}{*}{ S3 } & 0 & 46 & 57 & 57 & 58 \\
\hline & 1 & 11 & 0 & 1 & 0 \\
\hline & 2 & 0 & 1 & 0 & 0 \\
\hline & 3 & 1 & 0 & 0 & 0 \\
\hline
\end{tabular}

Number of lines missing years of data in datasets comprising bands $A-C, A-D, A-E$, and A-F. Total number of lines for selection: 638,176 , and 58 (for S1 to S3). 
TABLE 11 | Summary of environments in the chickpea MET dataset: number of trials for each stage of testing (S1, S2, S3) and total number of trials.

\begin{tabular}{|c|c|c|c|c|c|c|c|}
\hline \multirow[b]{2}{*}{ Environment } & \multicolumn{4}{|c|}{ Number of trials } & \multirow[b]{2}{*}{ nplot } & \multirow[b]{2}{*}{ nline } & \multirow{2}{*}{$\begin{array}{l}\text { Mean } \\
\text { yield }\end{array}$} \\
\hline & s1 & S2 & S3 & Total & & & \\
\hline 2016-Balaklava & 1 & 0 & 0 & 1 & 504 & 440 & 1.78 \\
\hline 2016-Horsham & 1 & 0 & 0 & 1 & 504 & 436 & 2.17 \\
\hline 2016-Melton & 1 & 0 & 0 & 1 & 504 & 450 & 2.17 \\
\hline 2016-Yenda & 1 & 0 & 0 & 1 & 504 & 360 & 2.59 \\
\hline 2017-Balaklava & 1 & 1 & 0 & 2 & 744 & 510 & 0.97 \\
\hline 2017-Curyo & 0 & 1 & 0 & 1 & 216 & 108 & 1.78 \\
\hline 2017-Horsham & 1 & 1 & 0 & 2 & 756 & 508 & 2.22 \\
\hline 2017-Melton & 1 & 1 & 0 & 2 & 756 & 512 & 1.36 \\
\hline 2017-Yenda & 1 & 1 & 0 & 2 & 924 & 648 & 1.18 \\
\hline 2017-York & 1 & 1 & 0 & 2 & 480 & 312 & 1.71 \\
\hline 2018-Ardlethan & 1 & 1 & 1 & 3 & 1356 & 972 & 0.66 \\
\hline 2018-Balaklava & 1 & 1 & 1 & 3 & 1032 & 676 & 0.44 \\
\hline 2018-Curyo & 0 & 1 & 1 & 2 & 492 & 210 & 0.74 \\
\hline 2018-Horsham & 1 & 1 & 1 & 3 & 948 & 599 & 2.02 \\
\hline 2018-Melton & 1 & 1 & 1 & 3 & 1032 & 676 & 0.49 \\
\hline 2018-Mingenew & 1 & 1 & 1 & 3 & 876 & 522 & 1.42 \\
\hline 2018-Northampton & 0 & 0 & 1 & 1 & 192 & 65 & 1.62 \\
\hline 2018-Wagga Wagga & 0 & 0 & 1 & 1 & 192 & 65 & 1.31 \\
\hline 2019-Ardlethan & 1 & 1 & 1 & 3 & 1260 & 858 & 0.35 \\
\hline 2019-Curyo & 0 & 1 & 1 & 2 & 444 & 180 & 1.63 \\
\hline 2019-Dalwallinu & 0 & 1 & 1 & 2 & 456 & 182 & 0.23 \\
\hline 2019-Horsham & 1 & 1 & 1 & 3 & 984 & 594 & 1.26 \\
\hline 2019-Melton & 1 & 1 & 1 & 3 & 816 & 414 & 0.59 \\
\hline 2019-Mingenew & 1 & 1 & 1 & 3 & 948 & 570 & 0.99 \\
\hline 2019-Narrabri & 1 & 0 & 0 & 1 & 780 & 642 & 2.11 \\
\hline 2019-Pinery & 1 & 1 & 1 & 3 & 756 & 368 & 0.78 \\
\hline 2019-Wagga Wagga & 0 & 0 & 1 & 1 & 240 & 81 & 0.58 \\
\hline 2019-Yenda & 0 & 0 & 1 & 1 & 240 & 80 & 1.38 \\
\hline
\end{tabular}

Number of plots (nplot) and lines (nline); mean yield (t/ha).

in $2019+$ S1 trials in 2018) and the final dataset. Note that for S1 selections, the single year dataset ( $\mathrm{S} 1$ trials in 2019) is equivalent to the band dataset (band A). The resultant A-values are shown in Figure 3. Once again the superiority of the final dataset in each case is clearly shown, with greatly reduced A-values compared to the single year and single band datasets.

\section{DISCUSSION}

In this paper we have addressed a void in the literature, and provided a rigorous framework for the construction of MET datasets for selection in plant breeding programs. We have described a method that aims to optimize the amount of information available on the "lines of interest," that is, the lines under consideration for selection. The method is intuitive and involves several simple steps. A key aspect is to identify contemporary groups (CGs), that is, groups of lines that entered

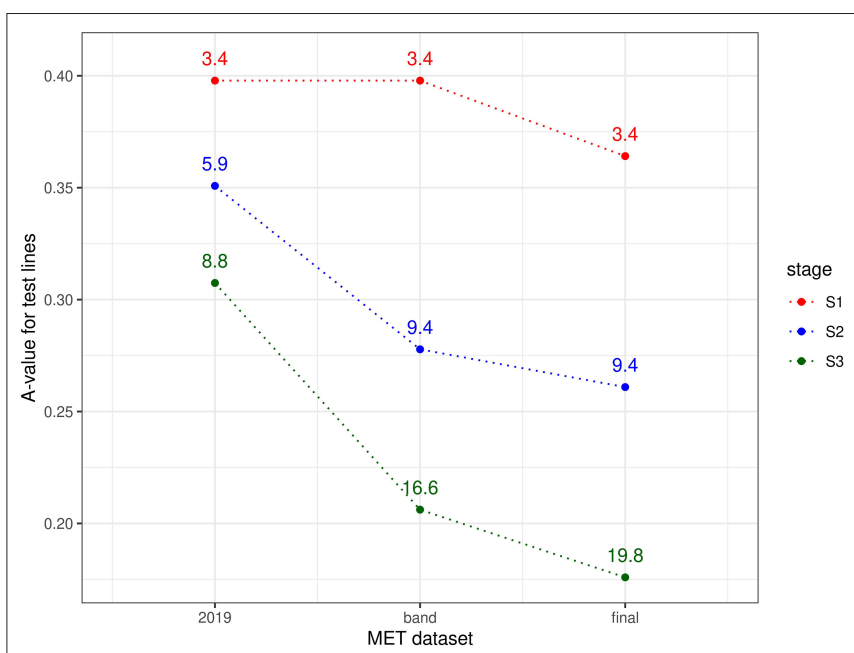

FIGURE 3 | A-values for lines under consideration for selection in chickpea breeding program in 2019 (stage S3: 58 lines, S2: 176 lines, and S1: 638

lines). A-values are given for three types of MET dataset, namely 2019 data for each stage; diagonal band of data for each stage and the final dataset constructed as in section 3.2. The points are labeled with the associated mean numbers of environments in which these lines were grown. Note that for S1

the 2019 and band datasets are the same.

the first stage of testing (S1) in the same year. This allows a complete enumeration of the trials in which the lines of interest have been grown. In addition to defining CGs, which relate to lines, we have also introduced the concept of data bands, which relate to trials. Data bands align with the testing system, namely the progression through stages (for example S1 through to S4) from year to year. By sequentially building the MET dataset using data bands we can form a dataset that captures as much information as possible for the lines of interest.

We have also developed a method for quantifying this information for any given MET dataset. The method uses fundamental concepts from model-based design theory. Thus, information is quantified using an A-value (average pairwise variance) for the lines of interest from a pre-specified linear mixed model. The application of this approach to the two motivating examples in this paper clearly showed the superiority of the MET datasets constructed using the CG approach, in particular when compared with the more common approach of simply using trials from a single stage and year. The information gains for all selection stages were associated both with direct information, that is, from the trials in which the lines of interest were grown and also indirect information derived from trials in which genetically related lines were grown.

MET datasets constructed using the CG approach encompass multiple bands of data and this suggests a further and crucial gain for S1 selection decisions since these lines have only been grown in a single year and often at only one or two locations. The key driver here is that locations with S1 trials also typically include later stage trials. The environment is then defined as the amalgamation of all of these (co-located) trials and thence includes a far wider set of lines than the S1 lines alone. This 
provides links between environments (both within and between years) for the S1 lines. Then application of the FALMM will allow examination of $\mathrm{G} \times \mathrm{E}$ for the $\mathrm{S} 1$ lines of interest across a wide range of environments. This is particularly important in an Australian context as genotype by location by year interaction is often the largest component of $\mathrm{G} \times \mathrm{E}$ (Frensham et al., 1998; Cullis et al., 2000). Hence the additional seasons of data will lessen the impact of selection on a single year of data, which could be a seasonally extreme year and therefore outside what is expected for the range of TPE. This issue has also been pointed out by Arief et al. (2015).

We note that combining trials utilizing the CG approach may result in an unbalanced dataset, with poor connectivity (low numbers of lines in common) between some environments. Whilst one of the advantages of using an FALMM is the ability to handle unbalanced data, there has been concern about the reliability of estimation of FA parameters in extreme cases when connectivity is very poor. It is well-known that poorly estimated genetic variance parameters will result in a reduction in genetic gain (Sales and Hill, 1976a,b). The A-value approach does not take this into account since the variance parameters are assumed known. Historically, genotype connectivity was thought to be the key determinant of the reliability of estimation of FA parameters. We are currently developing a formal information based diagnostic for this purpose. It is superior to connectivity in the sense of better forecasting the uncertainty of variance parameter estimates and being applicable for both additive and non-additive genetic variance parameters. It may therefore be applied jointly with the A-value approach in order to balance genotype information and reliability of variance parameter estimation in the search for an optimum MET dataset.

The applications in this paper are based on inbred annual crops. However, we note that our methods can be easily modified for situations that include hybrid crops, which require evaluation of inbred parental lines in addition to the hybrids themselves. The methods are also applicable for parental evaluation in perennial crops such as radiata pine, in which parental trees are evaluated by the performance of their progeny in (field) trials across numerous years and seasons (Smith and Cullis, 2018). We note that all models considered in this paper partitioned genetic effects into additive and non-additive effects. This was achieved with the use of a numerator relationship matrix formed from pedigree (ancestral) records. It would be straight-forward to replace this with a genomic relationship matrix formed from marker data.

Finally, and at the request of the editor, we note that an important area of research that requires MET datasets is the investigation of genotype by environment by management $(\mathrm{G} \times \mathrm{E} \times \mathrm{M})$ interactions. Our methodology was developed in the context of (standard) two-way $\mathrm{G} \times \mathrm{E}$ studies but could be generalized to three-way $\mathrm{G} \times \mathrm{E} \times \mathrm{M}$ studies using, for example, linear mixed models of the form presented in Smith et al. (2019). In Smith et al. (2019) the management practice was the application of a fungicide to canola seed in order to limit the impact of the disease blackleg. Within environments, the treatments comprised the factorial combinations of genotypes and the presence/absence of the fungicide and the experimental designs included replication for both factors. This allowed valid inference on $\mathrm{G} \times \mathrm{E} \times \mathrm{M}$ interactions. Unfortunately this is rarely the case for $\mathrm{G} \times \mathrm{E} \times \mathrm{M}$ studies, with the management treatment often not having proper replication. A common example is studies involving the presence/absence of an abiotic stress such as heat or drought. It is often argued that the nature of the equipment required for application of the stress factor is such that replication is not possible. This means that inference on $\mathrm{G} \times \mathrm{E} \times \mathrm{M}$ interactions is compromised. Kadkol et al. (2020) discuss this in detail. We argue that it should be possible to construct experimental designs that allow a limited, but sufficient amount of replication for stress treatments so that valid inference on $\mathrm{G} \times \mathrm{E} \times \mathrm{M}$ interactions is possible. Given the cost and importance of such research, this would seem a priority.

Our methodology for constructing a MET dataset has been programmed within the $\mathrm{R}$ computing environment ( $\mathrm{R}$ Development Core Team, 2015) and the code is available upon request. The method for quantifying information involves the use of ASReml-R (Butler et al., 2017) and example code is given in Appendix A. We have implemented these methods in a number of plant breeding programs with the result of superior MET datasets and also improved breeder confidence and understanding.

\section{DATA AVAILABILITY STATEMENT}

The datasets presented in this article are not readily available because they are owned by New South Wales Department of Primary Industries and the Grains Research and Development Corporation. Requests to access the datasets should be directed to gururaj.kadkol@dpi.nsw.gov.au (durum data) and kristy.hobson@dpi.nsw.gov.au (chickpea data).

\section{AUTHOR CONTRIBUTIONS}

BC and AS conceived the ideas and developed the methodology. AS wrote all sections of the manuscript apart from the Introduction which was written by AG. AG and CL prepared and curated the datasets. $\mathrm{GK}$ and $\mathrm{KH}$ provided the data and input on plant breeding perspectives. AS and CL performed the statistical calculations. BC and CL contributed to manuscript revision. All authors read and approved the submitted version.

\section{FUNDING}

AS was supported by the Grains Research and Development Corporation (GRDC) through the EssCargoT project (UW00010). The PBA Chickpea program received investment from GRDC, New South Wales Department of Primary Industries (NSW DPI), Queensland Department of Agriculture and Fisheries (DAF), Agriculture Victoria Research (AVR), and South Australian Research and Development Institute (SARDI).

\section{ACKNOWLEDGMENTS}

The authors thank the reviewers and editor whose comments have led to an improved manuscript. 


\section{REFERENCES}

Arief, V. N., DeLacy, I. H., Crossa, J., Payne, T., Singh, R., Braun, H.-J., et al. (2015). Evaluating testing strategies for plant breeding field trials: redesigning a CIMMYT international wheat nursery. Crop Sci. 55, 164-177. doi: 10.2135/cropsci2014.06.0415

Arief, V. N., Desmae, H., Hardner, C., DeLacy, I. H., Gilmour, A., Bull, J. K., et al. (2019). Utilization of multiyear plant breeding data to better predict genotype performance. Crop Sci. 59, 480-490. doi: 10.2135/cropsci2018.03.0182

Bänziger, M., and Cooper, M. (2001). Breeding for low input conditions and consequences for participatory plant breeding examples from tropical maize and wheat. Euphytica 122, 503-519. doi: 10.1023/A:1017510928038

Beeck, C., Cowling, W., Smith, A., and Cullis, B. R. (2010). Analysis of yield and oil from a series of canola breeding trials. Part I. Fitting factor analytic mixed models with pedigree information. Genome 53, 992-1001. doi: $10.1139 / \mathrm{G} 10-051$

Bernal-Vasquez, A.-M., Gordillo, A., Schmidt, M., and Piepho, H.-P. (2017). Genomic prediction in early selection stages using multi-year data in a hybrid rye breeding program. BMC Genet. 18:51. doi: 10.1186/s12863-0170512-8

Bueno Filho, J. S., and Gilmour, S. G. (2003). Planning incomplete block experiments when treatments are genetically related. Biometrics 59, 375-381. doi: 10.1111/1541-0420.00044

Butler, D. (2019). Pedicure: Pedigree Tools. R package version 2.0.0. Available online at: https://mmade.org/pedicure/

Butler, D. G., Cullis, B. R., Gilmour, A. R., Gogel, B. G., and Thompson, R. (2017). ASReml-R Reference Manual Version 4. Technical report, VSN International Ltd, Hemel Hempstead, United Kingdom.

Butler, D. G., Smith, A. B., and Cullis, B. R. (2014). On the design of field experiments with correlated treatment effects. J. Agric. Biol. Environ. Stat. 19, 539-555. doi: 10.1007/s13253-014-0191-0

Chapman, S., Cooper, M., Podlich, D., and Hammer, G. (2003). Evaluating plant breeding strategies by simulating gene action and dryland environment effects. Agron. J. 95, 99-113. doi: 10.2134/agronj2003.0099

Collard, B. C. Y., and Mackill, D. J. (2008). Marker-assisted selection: an approach for precision plant breeding in the twenty-first century. Philos. Trans. R. Soc. Lond. Ser. B Biol. Sci. 363, 557-572. doi: 10.1098/rstb.200 7.2170

Cullis, B., Gogel, B., Verbyla, A., and Thompson, R. (1998). Spatial analysis of multi-environment early generation variety trials. Biometrics $54,1-18$. doi: $10.2307 / 2533991$

Cullis, B., Jefferson, P., Thompson, R., and Smith, A. (2014). Factor analytic and reduced animal models for the investigation of additive genotype-byenvironment interaction in outcrossing plant species with application to a Pinus radiata breeding programme. Theor. Appl. Genet. 127, 2193-2210. doi: 10.1007/s00122-014-2373-0

Cullis, B., Smith, A., and Coombes, N. (2006). On the design of early generation variety trials with correlated data. J. Agric. Biol. Environ. Stat. 11, 381-393. doi: 10.1198/108571106X154443

Cullis, B. R., Smith, A., Beeck, C., and Cowling, W. (2010). Analysis of yield and oil from a series of canola breeding trials. Part II. Exploring variety by environment interaction using factor analysis. Genome 53, 1002-1016. doi: 10.1139/ G10-080

Cullis, B. R., Smith, A. B., Hunt, C., and Gilmour, A. R. (2000). An examination of the efficiency of Australian crop variety evaluation programmes. J. Agric. Sci. 135, 213-222. doi: 10.1017/S0021859699008163

Frensham, A., Barr, A., Cullis, B., and Pelham, S. (1998). A mixed model analysis of 10 years of oat evaluation data: use of agronomic information to explain genotype by environment interaction. Euphytica 99, 43-56. doi: 10.1023/A:1018395731621

Gilmour, A. R., Cullis, B. R., and Verbyla, A. P. (1997). Accounting for natural and extraneous variation in the analysis of field experiments. J. Agric. Biol. Environ. Stat. 2, 269-293. doi: 10.2307/1400446

Gogel, B., Smith, A., and Cullis, B. (2018). Comparison of a one-and two-stage mixed model analysis of Australia's National Variety Trial Southern Region wheat data. Euphytica 214:44. doi: 10.1007/s10681-0182116-4
Guilly, S., Dumont, T., Thong-Chane, A., Barau, L., and Hoarau, J.-Y. (2017). Analysis of multienvironment trials (MET) in the sugarcane breeding program of Réunion Island. Euphytica 213:213. doi: 10.1007/s10681-0171994-1

Kadkol, G., Smith, A., Cullis, B., and Chenu, K. (2020). Variation in Australian durum wheat germplasm for productivity traits under irrigated and rainfed conditions: genotype performance for agronomic traits and benchmarking. J. Agric. Sci. 1-17. doi: 10.1017/S0021859620000817

Moose, S. P., and Mumm, R. H. (2008). Molecular plant breeding as the foundation for 21st century crop improvement. Plant Physiol. 147, 969-977. doi: 10.1104/pp.108.118232

Oakey, H., Verbyla, A., Cullis, B., Wei, X., and Pitchford, W. (2007). Joint modeling of additive and non-additive (genetic line) effects in multi-environment trials. Theor. Appl. Genet. 114, 1319-1332. doi: 10.1007/s00122-007-0515-3

Perez-Elizalde, S., Jarquin, D., and Crossa, J. (2012). A general Bayesian estimation method of linear-bilinear models applied to plant breeding trials with genotype $\times$ environment interaction. J. Agric. Biol. Environ. Stat. 17, 15-37. doi: 10.1007/s13253-011-0063-9

R Development Core Team (2015). R: A Language and Environment for Statistical Computing. Vienna: R Foundation for Statistical Computing.

Redden, R., DeLacy, I., Butler, D., and Usher, T. (2000). Analysis of line $\mathrm{x}$ environment interactions for yield in navy beans. 2. Pattern analysis of lines and environment within years. Aust. J. Agric. Res. 51, 607-617. doi: 10.1071/AR97136

Rosielle, A., and Hamblin, J. (1981). Theoretical aspects of selection for yield in stress and non-stress environment. Crop Sci. 21, 943-946. doi: 10.2135/cropsci1981.0011183X002100060033x

Sabaghnia, N., Sabaghpour, S., and Dehghani, H. (2008). The use of an AMMI model and its parameters to analyse yield stability in multi-environment trials. J. Agric. Sci. 146:571. doi: 10.1017/S0021859608007831

Sales, J., and Hill, W. (1976a). The effect of sampling errors on the efficiency of selection indices. 1. Use of information on relatives for single trait improvement. Anim. Prod. 22, 1-17. doi: 10.1017/S0003356100035364

Sales, J., and Hill, W. (1976b). The effect of sampling errors on the efficiency of selection indices. 2. Use of information on associated traits for improvement of a single trait. Anim. Prod. 23, 1-14. doi: 10.1017/S0003356100031020

Smith, A., and Cullis, B. (2018). Plant breeding selection tools built on factor analytic mixed models for multi-environment trial data. Euphytica 214:143. doi: 10.1007/s10681-018-2220-5

Smith, A. B., Borg, L. M., Gogel, B. J., and Cullis, B. R. (2019). Estimation of factor analytic mixed models for the analysis of multi-treatment multi-environment trial data. J. Agric. Biol. Environ. Stat. 24, 573-588. doi: 10.1007/s13253-019-00362-6

Smith, A. B., Cullis, B. R., and Thompson, R. (2001). Analyzing variety by environment data using multiplicative mixed models and adjustments for spatial field trend. Biometrics 57, 1138-1147. doi: 10.1111/j.0006-341X.2001.01138.x

Stefanova, K. T., Smith, A. B., and Cullis, B. R. (2009). Enhanced diagnostics for the spatial analysis of field trials. J. Agric. Biol. Environ. Stat. 14, 392-410. doi: 10.1198/jabes.2009.07098

Thomason, W., and Phillips, S. (2006). Methods to evaluate wheat cultivar testing environments and improve cultivar selection protocols. Field Crops Res. 99, 87-95. doi: 10.1016/j.fcr.2006.03.007

Thompson, R. (1973). The estimation of variance and covariance components with an application when records are subject to culling. Biometrics 29, 527-550. doi: $10.2307 / 2529174$

Ukrainetz, N. K., Yanchuk, A. D., and Mansfield, S. D. (2018). Climatic drivers of genotype-environment interactions in lodgepole pine based on multienvironment trial data and a factor analytic model of additive covariance. Can. J. For. Res. 48, 835-854. doi: 10.1139/cjfr-2017-0367

Yan, W., and Rajcan, I. (2003). Prediction of cultivar performance based on single-versus multiple-year tests in soybean. Crop Sci. 43, 549-555. doi: $10.2135 /$ cropsci2003.0549

Yan, W., and Tinker, N. A. (2006). Biplot analysis of multi-environment trial data: principles and applications. Can. J. Plant Sci. 86, 623-645. doi: 10.4141/P05-169

Zamir, D. (2001). Improving plant breeding with exotic genetic libraries. Nat. Rev. Genet. 2, 983-989. doi: 10.1038/35103590 
Conflict of Interest: AG commenced employment with InterGrain when this manuscript was nearing completion.

The remaining authors declare that the research was conducted in the absence of any commercial or financial relationships that could be construed as a potential conflict of interest.
Copyright $\odot 2021$ Smith, Ganesalingam, Lisle, Kadkol, Hobson and Cullis. This is an open-access article distributed under the terms of the Creative Commons Attribution License (CC BY). The use, distribution or reproduction in other forums is permitted, provided the original author(s) and the copyright owner(s) are credited and that the original publication in this journal is cited, in accordance with accepted academic practice. No use, distribution or reproduction is permitted which does not comply with these terms. 


\section{APPENDICES}

\section{A. CODE FOR COMPUTING A-VALUES}

We consider S4 selection decisions for the final MET dataset for the durum motivating example. The data-frame is "final.df" and the key fields in this data-frame are "Environment" (factor with 30 levels); Gkeep (factor with 6,951 levels corresponding to the lines grown in the trials) and "Year" (factor with 6 levels). The inverse of the NRM is stored in sparse form in the object "durum.giv" and corresponds to 7,628 lines (lines grown in the trials and their ancestors). The mean of the diagonal elements of the NRM is $\bar{a}=1.9$. The steps in ASReml-R (Butler et al., 2017) are as follows:

1. run the model with start.values $=\mathrm{T}$ to be able to set the values of the variance parameters

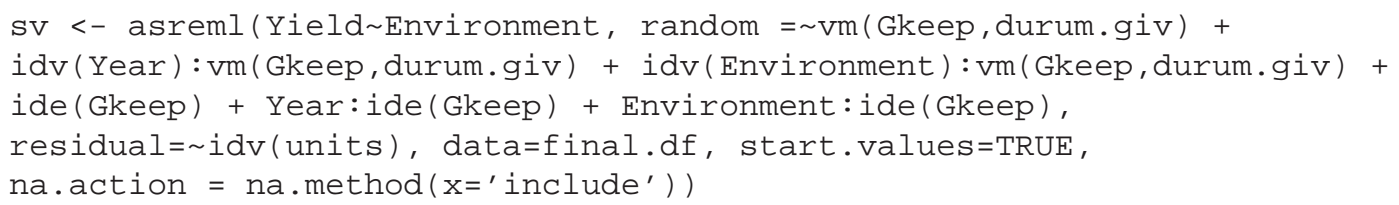

2. set the variance parameter values as given in Table 3. These are ordered as additive $G, G \times Y$ and $G \times E$ followed by non-additive $G$, $\mathrm{G} \times \mathrm{Y}$ and $\mathrm{G} \times \mathrm{E}$, then Error. Also set constraints so the parameters are fixed at these values in the next run of the model

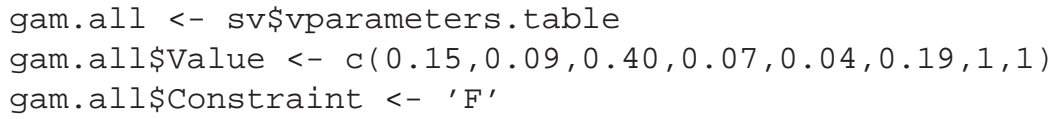

3. run the model with the (fixed) pre-specified variance parameter values

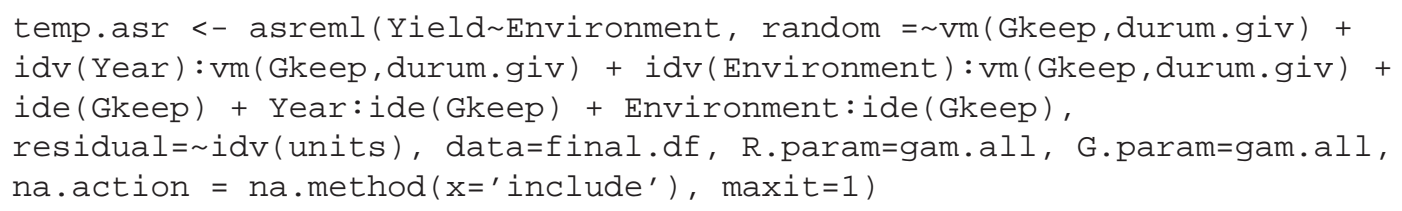

4. use the ASReml-R predict function to predict the total (additive + non-additive) line effects for the test lines in S4 in 2018 (there are 56 of these with names stored in the vector gs4.2018). Then obtain the A-value as the average pairwise variance (square of average sed)

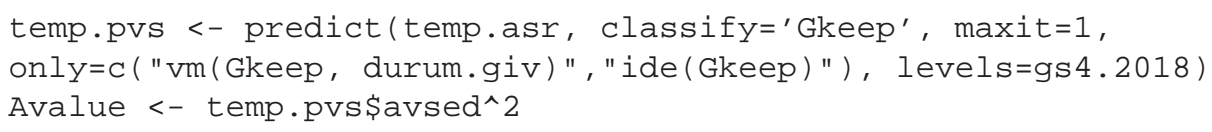

